\title{
$\beta$-Cyclodextrin-PANI decorated pencil graphite electrode for the electrochemical sensing of morin in almonds and mulberry leaves
}

\author{
Sherin Rison ${ }^{1,2} \cdot$ K. B. Akshaya ${ }^{2} \cdot$ Agnus T. Mathew $^{2} \cdot$ E. K. Joice ${ }^{1} \cdot$ Anitha Varghese $^{2}$ (I) $\cdot$ Louis George $^{2}$
}

Received: 12 May 2020 / Accepted: 17 September 2020 / Published online: 28 September 2020

(c) Springer Nature Switzerland AG 2020

\begin{abstract}
Morin (3,2,4,5,7-pentahydroxyflavone) is one of the natural flavonoids which is present in a variety of fruits and herbs. $\beta$-cyclodextrin ( $\beta-C D)$ and polyaniline (PANI) decorated Pencil graphite electrode (PGE) has been successfully used as a sensitive and conducting electrode for the determination of morin. The hydroxyl groups of $\beta-C D$ attract the analyte towards the modified electrode through hydrogen bonding. Cyclic voltammetry (CV) and electrochemical impedance spectroscopy (EIS) techniques were employed to study the electrochemical properties of the modified electrodes. The enhanced surface roughness of $\beta-C D-P A N I / P G E$ has resulted in the increase of electrocatalytic activity of electrode towards the analyte. Opitical profilometric studies were performed to evaluate the surface roughness of electrodes and differential pulse votammetry (DPV) was used for the quantitative analysis of morin. Scanning electron microscopy (SEM), Raman spectroscopy and Fourier transform infrared (FTIR) spectroscopy were carried out to know the physicochemical characteristics of the modified electrodes. The experimental conditions such as scan rate, $\mathrm{pH}$ and concentration were optimized. The electrochemical process was found to be adsorption controlled and irreversible from scan rate studies. Under optimal conditions, the linear dynamic range for the quantification of morin was found to be 1.17-32 nM. The low detection limit $(0.38 \mathrm{nM})$ indicates ultrasensitivity of the proposed method. The suggested method has been effectively employed for the determination of morin in almonds and mulberry leaves.
\end{abstract}

Keywords Pencil graphite electrode $\cdot$ Morin $\cdot \beta$-cyclodextrin $\cdot$ PANI $\cdot$ Electrooxidation

\section{Introduction}

Plants, fruits and flowers contain a variety of low molecular weight products that can strengthen the human immune and health system. They are rich in a variety of antioxidant molecules. Flavonoids are one of the antioxidant molecules that belong to a broad class of polyphenyl group containing benzene- $\gamma$-pyrone component. Morin (3,2,4,5,7-pentahydroxyflavone) is one of the most common natural flavonoids present in mulberry leaves, almonds, figs, grapes and some of the ayurvedic products [1, 2]. It can act as an antiviral, anti-inflammatory and anti-allergic compound in pharmaceutical industry [3]. It functions as a chemo preventive measure against oral carcinogenesis and protective measure for cardio activities [4]. The direct capturing of singlet oxygen family and poisoning of enzymes involved in the formation of reactive oxygen species elucidates the antioxidant feature of morin

Electronic supplementary material The online version of this article (https://doi.org/10.1007/s42452-020-03540-1) contains supplementary material, which is available to authorized users.

Anitha Varghese, anitha.varghese@christuniversity.in | ${ }^{1}$ Christ Academy Institute for Advanced Studies, Christ Nagar, Bangalore 560083, India. ${ }^{2}$ Department of Chemistry, CHRIST (Deemed to be University), Bangalore 560029, India. 
$[5,6]$. The structure of morin consists of two aromatic rings (Fig. S1) with an oxygen-containing heterocycle that connects ring $A$ and ring $B$. The hydroxyl groups at positions $2^{\prime}$ and $4^{\prime}$ in ring $B$ are the radical target sites of morin. The radical scavenging activity is also enhanced by hydroxyl groups at 5 and 7 positions of ring A. Hydroxyl groups at 3 and 4 ' positions are electrochemically oxidisable and form the respective quinones. Another key structural property of morin is the enhanced electron delocalization in ring $B$ resulting from the conjugation of double bond at 2, 3 positions with the keto group at position 4. UV-Vis absorption spectroscopy [7], spectrofluorimetry, HPLC [8], gas chromatography $[9,10]$ and electrochemical analysis have been reported for the determination of flavanoids. Among these methods, electrochemical analysis has achieved more attention due to its more economical, low energy consuming and unique approach in the method design, and high sensitivity for the analyte determination.

Literature study shows that not many electrochemical methods are available for the analysis of morin. Electrochemically prepared electrodes for determination of morin include vanadium pentoxide nanoflake film decorated glassy carbon electrode (GCE) [11], polyvinylpyrolidone (PVP) doped carbon paste electrode, Pt electrode [12], bare glassy carbon electrode (GCE) [13] and hanging mercury drop electrode [14], Ir decorated poly(3,4-ethylenedioxythiophene) on carbon fiber paper (Ir-PEDOT/ CFP) [15], 2,5-dimercapto-1,3,4-thiadazole modified carbon fiber paper (p-DMTD/CFP) [16] are also reported. An electrochemical sensing method with molecular imprinting mechanism using Pd nanoparticles-porous graphene carbon nanotubes (CNTs)/GCE has also been listed for quercetin [17]. But this method involves tiresome electrode preparation process.

Recently electrochemical properties of pencil graphite electrodes (PGEs) have been explored in the analysis of various organic compounds. In comparison to other working electrodes, PGE has lower background current, good adsorption properties, conductivity and ultra level electrochemical sensing ability towards different analytes [18-20]. Uniform composition and surface provided by PGE results in a good quality voltammetric signals of analytes. In 2018 H.T. Purushothama and co-workers developed a sensitive electrochemical sensor using PGE for the investigation of chlorpromazine, a phenothiazine class of drug [21]. Electro-activated pencil graphite electrode was used by David et al. for the voltammetric analysis of the flavonoid naringenin [22]. In 2019 S. Sharma and Co-workers published a review on PGE as a promising electrochemical sensor and I.G. David and co-workers in 2017 documented a study of role of PGE as an up-coming electroanalytical tool $[23,24]$. High electrochemical reactivity, easy modification, commercial accessibility, fine rigidity, disposability and low-cost of PGE make it ideal to be used as an effective working electrode.

Decoration of electrodes with polymeric materials by electropolymerization techniques is an efficient strategy in improving the functioning of electrochemical sensors. Polyaniline (PANI), polypyrrole (PPy), poly( 3,4 ethylenedioxythiophene) (PEDOT) and polythiophene (PTh) are a few well studied conducting polymers for the investigation of oxidation of various organic molecules $[25,26]$. Exceptional stability, ease of development, high electrical conductivity and redox properties are the characteristic features which make PANI a versatile conducting polymer in electrocatalytic applications [27]. Cyclodextrin (CD) is a macrocyclic glucose oligomer, consisting generally of six, seven or eight $D$ glucose units resulting in $\alpha-, \beta$ - or $\gamma-C D$, respectively $[28,29]$. Among them $\beta$-cyclodextrin has been successfully used for electrode modifications. Being a model host site, $\beta-C D$ is capable of allowing a variety of guest molecules inside their torus like designed cavities. The interaction of molecular cavities with guest molecules has been extensively analyzed with the intention of bringing more clarity to the specific recognition effects and selectivity in the reaction [30]. Zeng et. al reported a new method for $\beta-C D$ modified poly( $N$-acetylaniline) film electrode preparation by electrooxidation of poly $(\mathrm{N}$-acetyl anilne) (PNAANI) film in $\beta-C D$ [31]. The hydroxyl group, the nucleophile part of $\beta-C D$ can be attached to the polymer backbone. An electroactive probe, hydroquinone, was used to explore the $\beta$-CD/PNAANI film as the hydroquinone/quinone couple matches to the cavity of $\beta-C D$. Rahemi et. al created an electrochemical sensor for the determination of the chlorophenoxy herbicide 2-methyl4-chlorophenoxyacetic acid (MCPA) rooted on a blending of multi-walled CNTs with embodied $\beta-C D$ and PANI film modified GCE [32]. The high electrocatalytic efficiency of $\beta$-cyclodextrin and polyaniline film towards the oxidation of morin is yet to be investigated. In the present study PANI and $\beta-C D$ mixture has been used for the electrochemical modification of PGE in anionic micellar medium in presence of $\mathrm{H}_{2} \mathrm{SO}_{4}$. The developed electrode was effectively employed in electrochemical determination of morin in different real samples.

\section{Materials and methods}

\subsection{Reagents and materials}

Aniline, $\mathrm{KH}_{2} \mathrm{PO}_{4}, \mathrm{~K}_{2} \mathrm{HPO}_{4}$ and sodium dodecyl sulfate ( $\mathrm{SDS} \mathrm{C}_{12} \mathrm{H}_{25} \mathrm{NaOSO}_{3}$ ) were obtained from SD Fine chemicals Limited, India. Analytical grade $\mathrm{H}_{2} \mathrm{SO}_{4}, \beta-\mathrm{CD}$ and Morin were procured from Sigma Aldrich-Merck, India. Aqueous solutions were prepared using double distilled 
water for all the experimental studies. Camlin HB pencil graphite lead of $2 \mathrm{~mm}$ diameter and length of $1 \mathrm{~cm}$ was used as the electrode substrate for electrochemical studies.

\subsection{Apparatus}

Electrochemical analyzer, $\mathrm{CH} 180$ and CHI660E electrochemical workstation ( $\mathrm{CH}$ Instruments, Inc. USA) were used for CV and DPV measurements. The three electrode system used for the measurements consist of modified and bare PGE as working electrodes, platinum foil as the counter electrode and saturated calomel electrode (SCE) as the reference electrode. Scanning electron microscopy (SEM) analyses were carried out with FEI Company Scanning electron microscope Model Sirion. FTIR spectra was recorded on Thermo Nicolet, Avatar 370 instrument. Thermofisher Scientific Nicolet 6700 NXR FT- Raman module was used for FT- Raman analysis. HPLC analysis of morin was performed on a SHIMADZU LC-8A column $(250 \mathrm{~mm} \times 4.6 \mathrm{~mm})$ as the stationary phase.

\subsection{Preparation of modified electrode}

PGE of $0.65 \mathrm{~cm}^{2}$ was first pretreated with $1 \mathrm{M} \mathrm{H}_{2} \mathrm{SO}_{4}$ and washed. Electrical contact was given at one tip of the PGE using copper wire and silver conducting ink. The PGE edge was polished by making use of a sharp cutter and smoothened by emery paper.

PANI/PGE was made by the electropolymerization of aniline on PGE for 25 continuous scan cycles in the range $-0.6 \mathrm{~V}$ and $1.4 \mathrm{~V}$ at a scan rate $0.05 \mathrm{Vs}^{-1}$ using a solution containing $0.01 \mathrm{M}$ aniline, $0.01 \mathrm{M}$ sodium dodecyl sulfate and $0.1 \mathrm{M} \mathrm{H}_{2} \mathrm{SO}_{4}$. After deposition, the electrode was rinsed with water to eliminate excessively adsorbed aniline monomer and later dried. $\beta$-CD-PANI/PGE was developed electrochemically, using $0.01 \mathrm{M}$ aniline, $0.01 \mathrm{M}$ SDS and $0.1 \mathrm{M} \mathrm{H}_{2} \mathrm{SO}_{4} 0.01 \mathrm{M}$ of $\beta$-CD for 20 potential cycles at $0.05 \mathrm{Vs}^{-1}$ in the range -0.6 and $1.6 \mathrm{~V}$.

The behaviour of $\beta$-CD-PANI/PGE and similar electrodes in electrocatalytic oxidation of morin was explored by CV using a $50 \mathrm{~mL}$ glass cell containing $18 \mathrm{nM}$ morin in the potential range -0.2 and $1.4 \mathrm{~V}$ at $0.05 \mathrm{Vs}^{-1}$ scan rate in phosphate buffered solution (PBS) of $\mathrm{pH} 7.0$ as electrolyte. DPV measurements were carried out between $0-0.22 \mathrm{~V}$, for known concentrations of morin (within the linear dynamic range) with $0.05 \mathrm{~V}$ pulse amplitude, $0.06 \mathrm{~s}$ pulse width and $0.5 \mathrm{~s}$ pulse period. The anodic current of morin was measured at $0.12 \mathrm{~V}$ vs SCE and a calibration graph was constructed using anodic current against concentration of morin and used for the quantitative analysis.

\subsection{Extraction of morin from real samples}

Morus nigra L. (Raw mulberry leaves) were dried at $80^{\circ} \mathrm{C}$ for $2 \mathrm{~h}$. The dried leaves were powdered and $25 \mathrm{~g}$ of the powder was added to $50 \mathrm{~mL}$ of aqueous ethanol (1:1) and then filtered. The filtrate was rinsed with hexane and the aqueous part was separated using $\mathrm{CH}_{3} \mathrm{COOC}_{2} \mathrm{H}_{5}$. It was then allowed to dry and the formed residue was dissolved in ethyl alcohol. The obtained solution was used for the analysis of morin. Morin was analyzed by DPV using standard addition method and the morin content was determined for $1 \mathrm{~g}$ of raw mulberry leaves.

Almonds were procured from a nearby grocery store. The outer cover of almonds were removed and dried at 25 ${ }^{\circ} \mathrm{C}$ in the absence of sunlight. Almond hulls were ground in a mill before the extraction process. For the extraction with ethanol $(70 \%)$ /water (30\%) mixture, $2 \mathrm{~g}$ of the sample was suspended in $20 \mathrm{~mL}$ of the solvent mixture and filtered using Whatman No. 4 paper. The solvents were evaporated and the aqueous extracts obtained were dissolved in water and analysed by using the proposed procedure [33]..

\section{Results and discussion}

\subsection{Electrochemical preparation of $\beta$-CD-PANI/PGE}

The cyclic voltammetry result obtained for the electropolymerization of aniline on PGE, from an electrolyte consisting of $0.01 \mathrm{M}$ aniline, $0.01 \mathrm{M}$ sodium dodecyl sulphate and $0.1 \mathrm{M} \mathrm{H}_{2} \mathrm{SO}_{4}$ for 25 cycles at a sweep rate of $0.05 \mathrm{Vs}^{-1}$ between the potential range $-0.6 \mathrm{~V}$ and $1.4 \mathrm{~V}$ is shown in Fig. S2a. The solubility of aniline in acidic aqueous media is enhanced by an anionic surfactant SDS which resulted in an increased rate of electrochemical polymerization. The anodic peak of aniline begins at $0.33 \mathrm{~V}$ in the first cycle and produces the first layer of polyaniline. The progressive growth of PANI film is reflected from the improved redox current in successive scans. Two redox couples were found at $0.335 \mathrm{~V}$ and $-0.002 \mathrm{~V} ; 0.670 \mathrm{~V}$ and $0.438 \mathrm{~V}$ in the voltammograms.

The mechanism of formation of PANI by electrochemical polymerization involves the formation of radical cations leading to dimerization. The oligomers formed in the propagation step endure nucleation, leading to the growth of polymer molecule (Fig S3) [34].

Fast electron transfer between morin and electrode is facilitated by $\beta$-CD-PANI modified PGE. The modified electrode was prepared by the electrodeposition of aniline and $\beta-C D$ in presence of $\mathrm{SDS}$ and $\mathrm{H}_{2} \mathrm{SO}_{4}$ as supporting electrolyte. The hydroxyl groups present in $\beta-C D$ attract morin molecules towards the electrode surface due to the strong electrostatic interaction. (Fig. S2b). The strong hydrogen 
bond between oxygen atom of hydroxyl group and $-\mathrm{NH}$ group on aniline makes $\beta$-CD-PANI modification on PGE more stable. The physicochemical studies prove the presence of hydrogen bonding in $\beta$-CD-PANI/PGE.

The PGEs modified with $\beta-C D$ and PANI were washed with double distilled water, dried and used for anodic oxidation of morin. To study the electrodeposition of $\beta-C D$ and PANI on PGE for the electrocatalytic oxidation of morin, different potentials scanning rates were applied for a fixed concentration of morin.

\subsection{Voltammetric behavior of $\mathrm{K}_{4}\left[\mathrm{Fe}(\mathrm{CN})_{6}\right] /$ $\mathrm{K}_{3}\left[\mathrm{Fe}(\mathrm{CN})_{6}\right]$ at $\beta-\mathrm{CD}-\mathrm{PANI} / \mathrm{PGE}$ and PANI/PGE}

The electrochemical response of ferrocyanide/ferricyanide at $\beta$-CD -PANI/PGE, PANI/PGE and bare PGE were analysed at $0.05 \mathrm{~V} / \mathrm{s}$ (Fig. S4). The electroactive surface area of the electrode was estimated from the cyclic voltammetric data acquired for the redox couple. The oxidation peak current was plotted against square root of scan rate and slope was determined from the resultant graph. Randles-Sevcik equation (Eq. 1) was employed to compute the surface area of the electrode from the slope value, concentration and diffusion coefficient $\left((6.70 \pm 0.02) \times 10^{-6} \mathrm{~cm}^{2} / \mathrm{s}\right)[35]$.

$\mathrm{i}_{\mathrm{p}}=2.69 \times 10^{5} A D_{0}^{1 / 2} \mathrm{n}^{3 / 2} v^{1 / 2} \mathrm{C}$

where $A$ stands for electroactive surface area in $\mathrm{cm}^{2}, D_{0}$ represents the diffusion coefficient in $\mathrm{cm}^{2} / \mathrm{s}, \mathrm{n}$ indicates the number of electrons participating in the redox reaction $(n=1), v$ is the scan rate in $V / s$, and $C$ is the concentration of $\mathrm{K}_{4}\left[\mathrm{Fe}(\mathrm{CN})_{6}\right] / \mathrm{K}_{3}\left[\mathrm{Fe}(\mathrm{CN})_{6}\right]$ in the solution in mol/ $\mathrm{cm}^{3}$. The surface area calculated for bare PGE, PANI/PGE, and $\beta$-CD-PANI/PGE were found as 1.121, 2.225 and 2.710 $\mathrm{cm}^{2}$ respectively. The calculated surface area value of bare PGE was found lower than that of modified electrodes. These results revealed that $\beta$-CD-PANI thin layer provides enhanced surface area to PGE.

\subsection{Physicochemical characterization of modified electrode}

\subsubsection{SEM}

SEM images of bare PGE, PANI/PGE and $\beta$-CD-PANI/PGE are shown in Fig. 1. The Fig. $1 \mathrm{~b}$ confirms the elctrodeposition of polyaniline on the bare substrate. The electrochemically deposited polyaniline increased the electroactive surface area and PGE substrate was uniformly covered by the polymer. The presence of surfactant provided the support for the uniform development of polyaniline on substrate electrode. The SEM image (Fig. 1c) clearly explains the high porous nature and increased surface defects of the $\beta$-CD-PANI/PGE compared to PANI/PGE and bare PGE. The $\beta$-CD-PANI/PGE enhanced the electrode electrolyte contact area by increasing the electroactive sites. It also provides a greater contact time for the morin molecules to get easily oxidized.

\subsubsection{Raman and IR spectroscopic studies}

The IR spectra of pure PANI and $\beta$-CD-PANI nanoclustres are presented in Fig. S5. In IR spectrum of PANI the peak at $1583 \mathrm{~cm}^{-1}$ shows $C=C$ stretching of the quinonoid ring and peak at $1490 \mathrm{~cm}^{-1}$ implies $C=C$ stretching of the benzenoid ring. The asymmetric vibration of amine group is observed at $3325 \mathrm{~cm}^{-1}$. The signal at $2922 \mathrm{~cm}^{-1}$ stands for aromatic sp ${ }^{2} \mathrm{CH}$ stretching. Peaks observed at 1304, 1142 and $827 \mathrm{~cm}^{-1}$ are due to $\mathrm{C}-\mathrm{N}$ stretching of secondary amine, $\mathrm{C}=\mathrm{N}$ stretching and ring vibrations of emaraldine salts of PANI. In the IR spectra of $\beta$-CD-PANI, the peaks corresponding to hydroxyl groups present on $\beta-C D$ and amine group of PANI got merged, broadened and shifted to lower frequency region due to hydrogen bonding. All other peaks remain the same having slight shifts in their position. Raman spectra (Fig. S6) clearly shows the two significant peaks at 1583 and $1352 \mathrm{~cm}^{-1}$ for both PANI/PGE
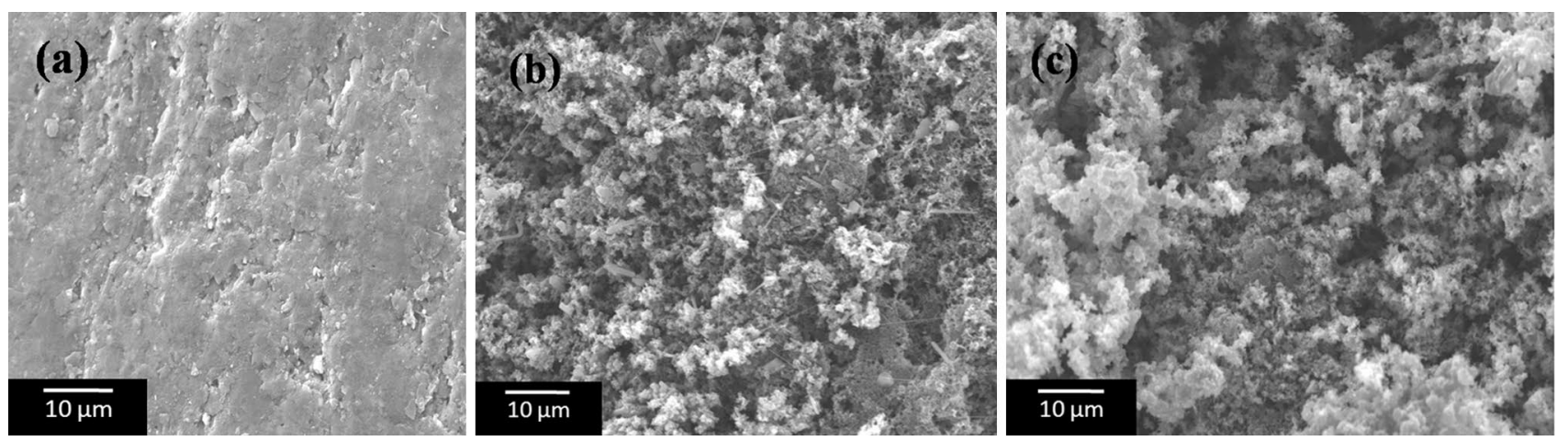

Fig. 1 SEM images of bare PGE (a), PANI/PGE (b) and $\beta-C D-P A N I / P G E(\mathbf{c})$

SN Applied Sciences

A SPRINGER NATURE journal 
and $\beta$-CD-PANI/PGE which explains $G$ band and $D$ band of $\mathrm{sp}^{2}$ hybridized carbon atoms of PGE. The $\mathrm{CN}^{+}$stretching is explained by $1209 \mathrm{~cm}^{-1}$ peak. The peak observed at $1530 \mathrm{~cm}^{-1}$ indicates $C-C$ stretching of benzenoid ring. The band at $1583 \mathrm{~cm}^{-1}$ is also attributed to the $C=N$ mode of quinonoid rings. The increase in peak intensity for $\beta-C D$ PANI/PGE is due to the increase in substantial amount of irregularities on the electrode surface.

\subsubsection{Optical profilometric studies of bare PGE and modified $\beta-C D-P A N I / P G E$}

The surface morphology and roughness of bare Pencil graphite electrode and modified $\beta-C D-P A N I / P G E$ were analyzed using optical profilometric studies. $2 \mathrm{D}$ and $3 \mathrm{D}$ images taken from optical profilometer are given in Fig. 2 and Fig. S7. As evident from Fig. 2a, 2b and Fig. S7a, S7b, bare PGE showed morphology of planar surface of graphite. Root mean square roughness of bare PGE was found as $1.7412 \pm 0.3015 \mu \mathrm{m}$. Figure $2 \mathrm{c}$, S7c and Fig. $2 \mathrm{~d}$, S7d exhibit $2 \mathrm{D}$ image and 3D profile of PGE deposited with PANI and $\beta$-cyclodextrin ( $\beta-C D)$ together respectively. It reveals that the optical characteristics of the surface is not same after coating with PANI and $\beta-C D$. Besides, the roughness of the surface was seemed to raise notably to $3.7902 \pm 1.5259 \mu \mathrm{m}$ after the deposition of PANI and $\beta-C D$ which denotes the development of a rough film of $\beta$-CD-PANI on bare PGE.
This spike in roughness can be attributed to the formation of small dark shaded spherically shaped particles of $\beta-C D$ and PANI on bare PGE. Electrocatalytic performance of these electrodes are directly correlated to the roughness of their surfaces. The surface having highest roughness ( $\beta$-CD-PANI/PGE) exhibited elevated electrocatalytic efficiency towards morin detection. Higher surface roughness caused to increase the electroactive area which resulted in to step up the catalytic activity of the modified electrodes.

\subsection{Evaluation of modified electrodes by electrochemical impedance spectroscopy (EIS)}

EIS is an effective method to characterize the interface characteristics of electrodes and is generally employed in the characterization of modified electrodes. Fig. $\mathrm{S} 8$ reveals Nyquist plots of $\beta$-CD-PANI/PGE (Fig. S8b), PANI/PGE (Fig. S8a) and bare PGE (inset) in $1 \mathrm{mM} \mathrm{K}_{4}\left[\mathrm{Fe}(\mathrm{CN})_{6}\right] / \mathrm{K}_{3}\left[\mathrm{Fe}(\mathrm{CN})_{6}\right]$ in $1 \mathrm{M} \mathrm{KCl}$. The semicircular diameter (charge transfer resistance, $R_{c t}$ ) of Nyquist plot of bare PGE was found to be $184.5 \Omega$, indicating a high $R_{c t}$ value. This implies weak conductivity and penurious sensitivity of PGE, whereas the diminished $R_{c t}$ value of $86.98 \Omega$ with the modification with PANI, confirming the enhanced conductance. $\beta$-CD-PANI modification on PGE resulted in further decrease of $R_{c t}$ value $(2.949 \Omega$ ) which confirms the increase in conductivity
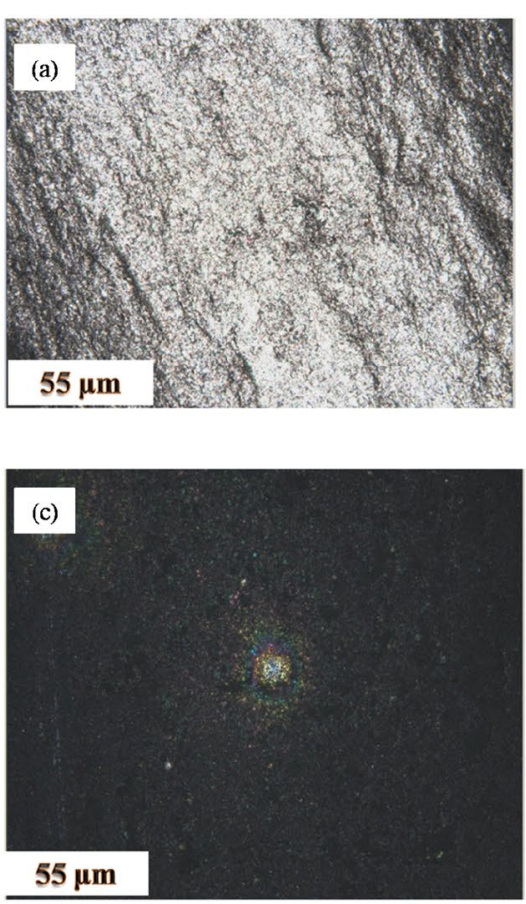
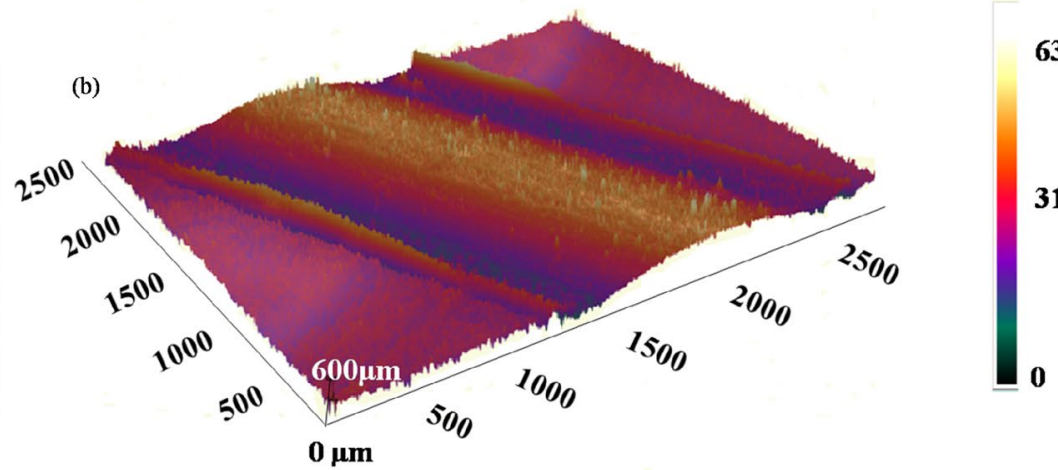

$630.72 \mu \mathrm{m}$

314.12

(d)

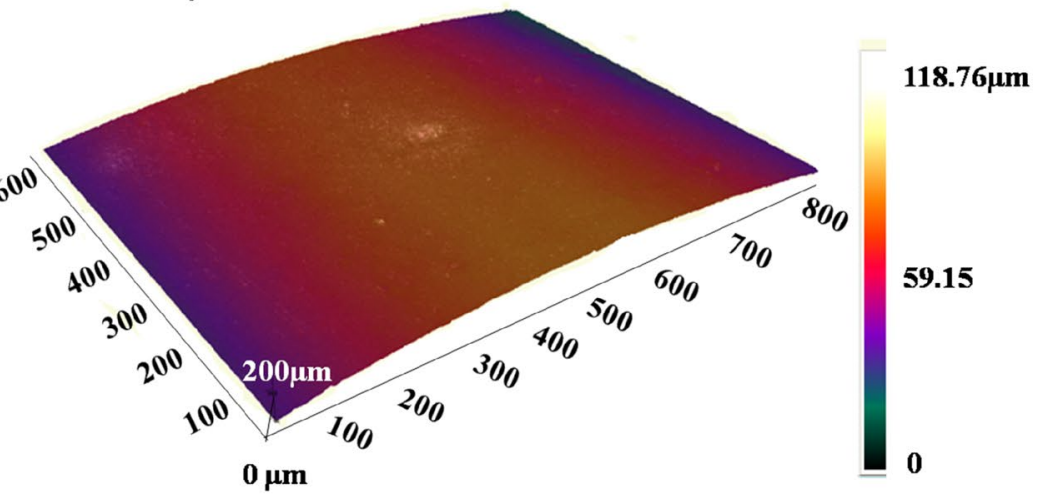

Fig. 2 Optical profilometeric images: 2D and 3D images at higher magnification $20 \times$ of Bare PGE (a and b), $\beta$ CD-PANI/PGE (c and d) 
of $\beta$-CD-PANI/PGE. The modification of bare PGE by $\beta-C D$ and PANI intensify the conductivity of electrode towards the electrocatalytic oxidation of $\mathrm{K}_{4}\left[\mathrm{Fe}(\mathrm{CN})_{6}\right] / \mathrm{K}_{3}\left[\mathrm{Fe}(\mathrm{CN})_{6}\right]$. A rise in slope of linear portion (Warburg impedance) in the graph denotes substantial diffusion activity of ferrocyanide/ferricyanide towards $\beta$-CD-PANI/PGE. These results confirm the potential of the developed electrode

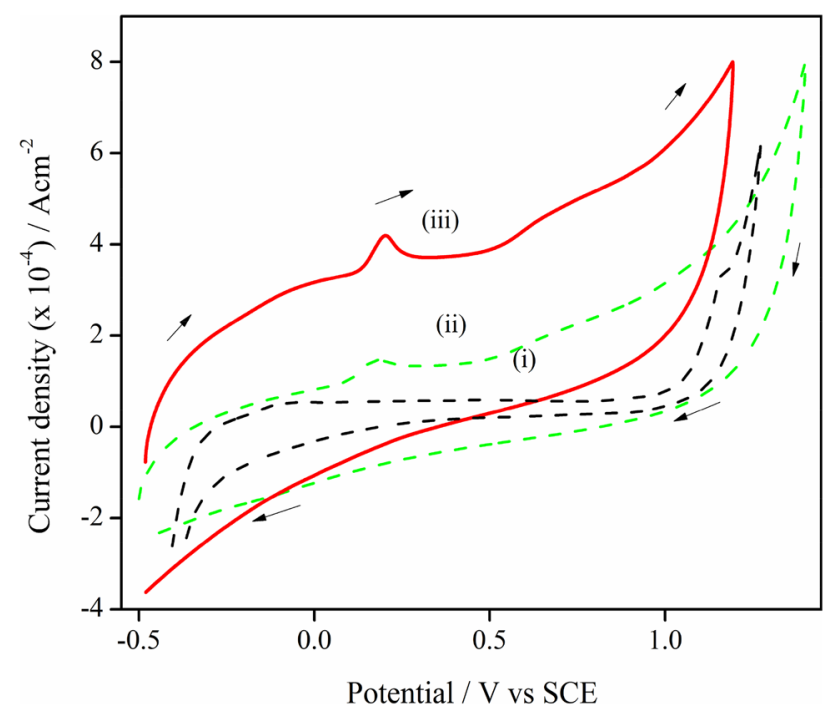

Fig. 3 Cyclic votammogram of $18 \mathrm{nM}$ morin at (i) bare PGE (ii) PANI/ PGE (iii) $\beta$-CD-PANI/PGE in PBS pH 7.0 to stage an excellent platform for electrocatalytic oxidation of morin.

\subsection{Electrocatalytic Oxidation of morin on $\beta$-CD-PANI/GPE}

Cyclic voltammetric studies were conducted to investigate the electrocatalytic oxidation of morin at $\beta-C D-P A N I / P G E$, PANI/PGE and bare PGE. The experiment was conducted at a scan rate of $0.05 \mathrm{Vs}^{-1}$ in PBS (pH 7.0). A low current flow was observed for bare PGE and PANI/PGE electrodes (curves 'i \& ii') where as a wide voltammogram (curve 'iii') was obtained for $\beta-C D$-PANI/PGE during the electrocatalytic oxidation of morin ( $0.2 \mathrm{~V}$ ) (Fig. 3). The high energy surface active sites formed by $\beta-C D$ and PANI deposition on substrate electrode strongly attracted the molecules of morin towards the surface. The large number of hydroxyl groups present in both $\beta$-CD-PANI/PGE and analyte also favors the attraction of morin towards the modified electrode. Schematic representation of electrocatalytic oxidation of morin at $\beta-C D$-PANI/PGE is given in the Fig. 4.

\subsection{Influence of scan rate on morin oxidation}

The cyclic voltammograms of $18 \mathrm{nM}$ morin at $\beta$-CD-PANI/ PGE at various scan rates between $0.02 \mathrm{~V} / \mathrm{s}$ and $0.15 \mathrm{~V} / \mathrm{s}$ in PBS ( $\mathrm{pH}$ 7.0) medium are shown in Fig. S9. The anodic peak current $\left(\mathrm{i}_{\mathrm{pa}}\right)$ was found to be raised linearly with the scan rate. (Fig. S10). It proves that an adsorption controlled

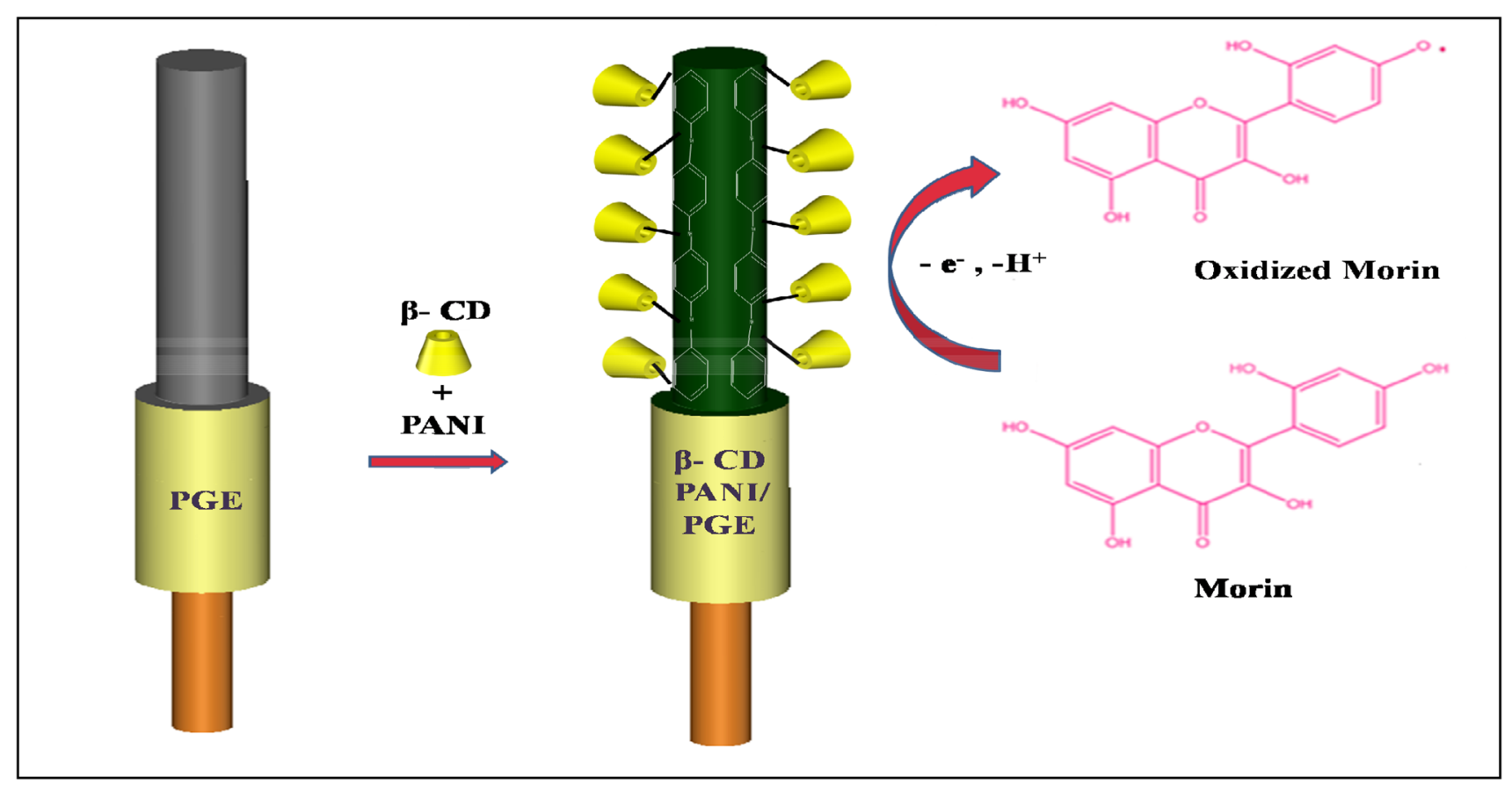

Fig. 4 Schematic representation of electrocatalytic oxidation of morin at $\beta-C D$-PANI/PGE 
reaction has occurred at the electrode surface. The kinetics of electrode surface reaction was determined by investigating the effect of various scan rates $(v)$ on anodic peak currents. A straight line graph was generated when logarithm of peak currents were plotted against the logarithm of scan rates (Fig. S11). Theoretically the slope of an adsorption controlled process is one. The slope value (0.9820) obtained from the calibration plot which is near to the theoretical value revealed that the electrode has undergone an adsorption controlled process.

$\log i_{p a}(\mu A)=0.9820 \log v\left(V^{-1}\right)+3.7989 ;\left(R^{2}=0.9976\right)$

The number of electrons involved in the electrocatalytic oxidation of morin estimated using the equation given below was found to be approximately 2 .

$E p-E p / 2=0.0564 / n$

where $\mathrm{Ep} / 2$ and Ep indicates half peak potential and peak potential.

Further, from the slope of $E$ vs log $u$ graph the value was obtained as 0.0619 . The slope is equivalent to $2.303 \mathrm{RT} /$ anF, where $\mathrm{n}$ is the number of electrons, $\mathrm{R}$ is gas constant, $\mathrm{T}$, temperature, $\mathrm{F}$ is faraday constant and a (electron transfer coefficient) is usually 0.5 [36]. For the determination of morin the value of $\mathrm{n}$ is obtained as 1.91 , which is approximately equal to 2 . The linear regression equation for $E$ vs $\log u$ is given below.

$\mathrm{E}_{\mathrm{pa}}(\mathrm{V})=0.0619 \log v\left(\mathrm{~V} \mathrm{~s}^{-1}\right)+0.3812 ;\left(\mathrm{R}^{2}=0.9913\right)$

From Laviron's equation the electrochemical adsorption of morin on the modified electrode obeys Langmuir absorption isotherm when the $i_{p a}$ has a linear relationship with $u$ [37].

$\mathrm{i}_{\mathrm{pa}}=\mathrm{nFQ} v / 4 \mathrm{RT}$

where $\mathrm{n}$ is number of electrons and $\mathrm{Q}$ is the charge involved. From the calculation the number of electrons was found to be 2.12 which is validated by Laviron's theory and approximated at 2 .

\subsection{Effect of number cycles of $\beta$-CD-PANI coating on electrocatalytic oxidation of morin}

Effect of the number of cycles necessary for coating of $\beta$-CD-PANI over PGE on the electrochemical determination of morin was studied. $\beta$-CD-PANI/PGEs were prepared by using different scanning potential cycles such as 2,4 , $6,8,10,12,14,16,18$ and 20 and their efficiency towards oxidation of $18 \mathrm{~nm}$ Morin in $\mathrm{pH} 7.0$ was investigated. Cyclic voltammograms for the detection of morin with respective modified electrode is compared in Fig. S12. Oxidation peak current of analyte was found to be increasing with increase in number of cycles for the electro-deposition of $\beta-C D$ PANI on PGE. The optimized scanning cycles for $\beta$-CD-PANI deposition was taken as 20 cycles as the anodic current continued to be constant after 20 cycles.

\subsection{Optimization of $\mathrm{pH}$}

The influence of $\mathrm{pH}$ from 2 to 12 on anodic peak current of morin (18 nM) in PBS was studied. Fig. S13 indicates that there is an increase of oxidative peak currents upto $\mathrm{pH} 7.0$ and further change of $\mathrm{pH}$ range followed a slow and steady drop in current. The result of $\beta-C D-P A N I / P G E$ was not significant at lower $\mathrm{pH}$ range, due to a reduction in electron charge density of $\beta$-CD-PANI while at elevated range of $\mathrm{pH}$, the anodic current reduced owing to the partial oxidation of the substrate by dissolved molecular oxygen. Therefore, PBS with $\mathrm{pH} 7.0$ was selected for the present study. A positive peak potential shift was observed in the graph of $\mathrm{pH}$ against anodic peak potential (Fig. S14) as the $\mathrm{pH}$ varies from 2 to 12 . This can be shown as a linear regression equation.

Epa $(V)=0.005+0.05926 \mathrm{pH}\left(\mathrm{R}^{2}=0.9926\right)$

A slope of $0.05926 \mathrm{~V} / \mathrm{pH}$ was observed to be in near conformity with the theoretical value of $0.059 \mathrm{~V} / \mathrm{pH}$, thus proposing participation of same number of protons and electrons in the reaction. Therefore, PBS with $\mathrm{pH} 7.0$ was selected for the present study.

\subsection{Differntial pulse voltammetric studies}

The linear dynamic range, limit of detection (LOD) and limit of quantitation (LOQ) were obtained for the quantification of the analyte under study. DPV method was adopted for this purpose and the oxidative peak currents were measured at $0.12 \mathrm{~V}$ (Fig. S15). According to Fig. S16, the anodic peak current is observed to increase with rise in concentration of morin. The linear calibration graph for the quantification of morin is given in Fig. S16. The linear regression equation is as shown below,

$\mathrm{i}_{\mathrm{pa}}(\mathrm{mA})=5.4773 \mathrm{E}^{-7}[$ Morin $]-1.993 \mathrm{E}^{-4}\left(\mathrm{R}^{2}=0.9984\right)$

The sensitivity of the process was evaluated as

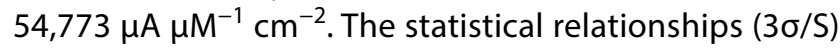
and $(10 \sigma / \mathrm{S})$ are employed to calculate the LOD and LOQ respectively, where $\sigma$ stands for the standard deviation of the peak current at any given analyte concentration and $S$ indicates the slope of the calibration curve. The LOD for the determination of morin was estimated as $0.38 \mathrm{nM}$ and the LOQ $1.17 \mathrm{nM}$ which was used to determine the lower limit of linear dynamic range. Linear dynamic rage was 
found to be 1.17-32 $\mathrm{nM}$. The value of LOD obtained for $\beta-C D-P A N I / P G E$ is so far the best documented value for quantitative analysis of morin which can be ascribed to the high sensitivity of $\beta$-CD-PANI/PGE.

\subsection{Interference study}

The impact of interfering molecules against current densities in Morin detection (18 $\mathrm{nm}$ ) is presented as bar graphs in Fig. S17. Inorganic cations like $\mathrm{Na}+, \mathrm{K}^{+}, \mathrm{Mg}^{2+}$ and $\mathrm{Ca}^{2+}$ with concentration 440 folds that of morin had no interference in the analysis of analyte. Simiarly anions such as $\mathrm{Cl}^{-}$, $\mathrm{NO}_{3}{ }^{-}$and $\mathrm{CO}_{3}{ }^{2-}$ also did not have interference in morin analysis to the extent of 320 folds concentration of morin. Futhermore, molecules such as thearubigins, catechins and theaflavins with closely related structures have not interfered in the determination of morin. But, flavanoids like quercetin and rutin with similar structure as morin have exhibited anodic peaks at higher potential values with very low current density. Hence, the determination of morin is having only very negligible interference from these groups of compounds. Table S1 shows a list of potential interferences from some species on the voltammetric response in the determination of morin. The low relative standard deviation values for the analysis of five replicates of morin in presence of interfernts indicated good precision of the proposed method. Thus, the present method is highly efficient for the electrocatalytic determination of morin in real sample matrices.

\subsection{Reproducibility and stability}

Electrochemical response of five modified electrodes towards morin ( $18 \mathrm{~nm}$ ) oxidation was investigated and the parameters are given in Table S2. The results indicate the high reproducibility of the modified electrodes. All the 5 electrodes were also tested towards the oxidation of morin at an interval of five days for one month. The results indicated the long term stability of electrode and the decrease in $C V$ response of the electrode was less than $4.5 \%$. $\beta-C D$ PANI/PGE was subjected to 50 continuous potential scanning cycles in PBS ( $\mathrm{pH} 7.0$ ) and established the stability of the modified electrode (Fig. S18).

\subsection{Determination of morin in almonds and mulberry leaves}

The feasibility of the suggested technique was analyzed by determining the analyte, morin in almonds and mulberry leaves. Differential Pulse Voltammetry was adopted to check the extraction efficiency. Experimental factors like extraction order, extraction time and volume of extractant were optimized. In DPV studies of all real samples a

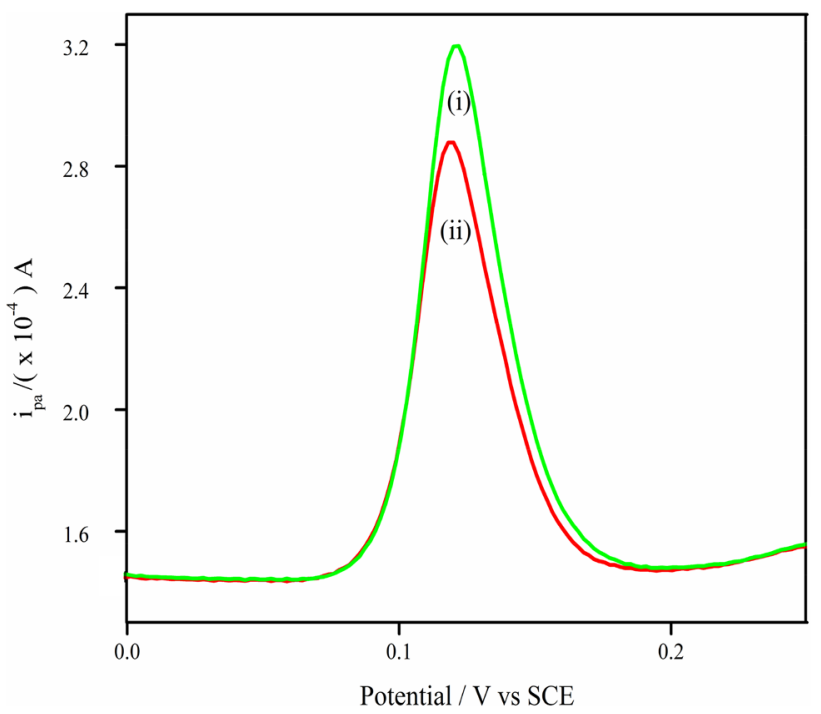

Fig. 5 DPVs of morin in real samples (i) mulberry leaves and (ii) almonds in the potential range $0 \mathrm{~V}-0.22 \mathrm{~V}$ vs SCE in PBS pH 7.0

significant anodic peak at $0.12 \mathrm{~V}$ was observed (Fig. 5). The reproducibility of the proposed method with $\beta$-CD-PANI/ PGE was also investigated by analyzing morin in spiked samples having the extracts of almonds and mulberry leaves. The results revealed that the developed method was competent in the electrochemical determination of morin in real samples (Table 1). Statistical analysis of the results by Student's t-test and variance ratio F-test showed no significant difference in accuracy and precision between proposed and standard reference method (HPLC) [9]. The result is included in Table 1. The recovery values varied from $95.9 \%$ to $99.6 \%$. Table S3 gives the comparative study of analytical parameters of suggested method for the analysis of morin with the reported works.

\section{Conclusion}

$\beta$-CD-PANI modified pencil graphite electrode exhibited good electrocatalytic activity towards oxidation of morin which is favored by electrostatic interaction between hydroxyl groups of morin and $\beta$-cyclodextrin which is strongly bound to PANI film through hydrogen bonding. The irreversable, adsorption controlled electrooxidation of morin is found to be one step reaction involving two electrons. Low detection limit, high sensitivity and selectivity are the major highlights of the proposed method over the other methods exist in the literature. The developed sensor has shown high reproducibility and stability. The proposed method was successfully employed for the analysis of morin in almonds and mulberry leaves. 


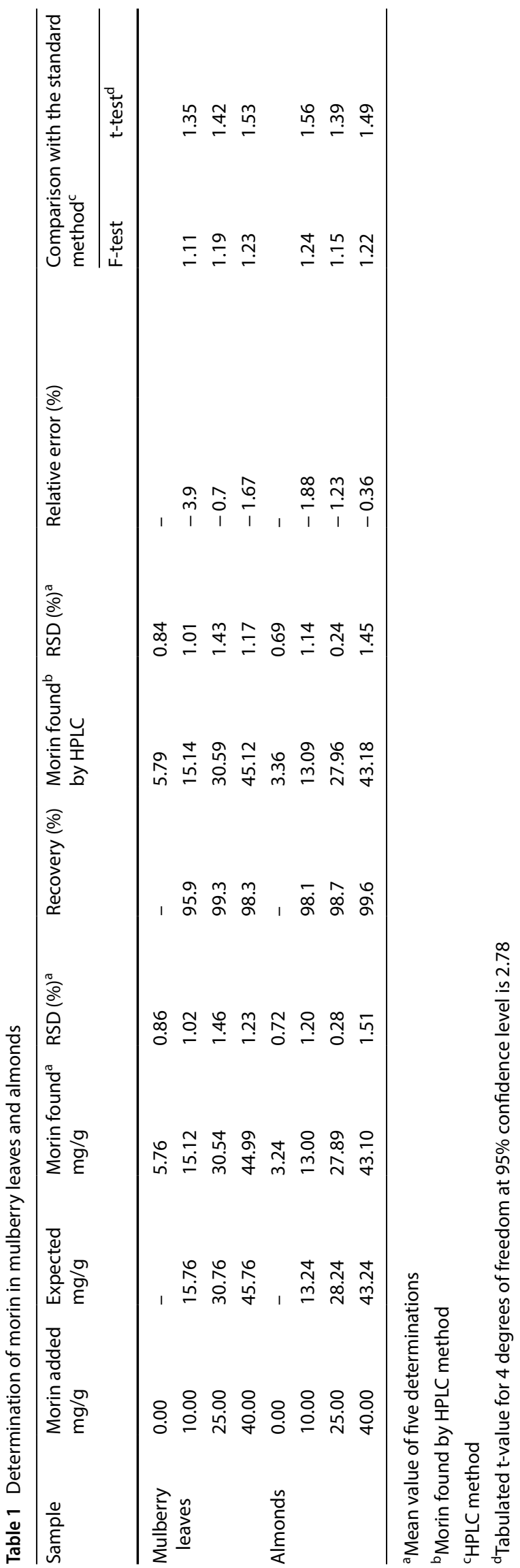

Acknowledgements The authors are thankful to IISC, Benguluru for providing Raman spectroscopic and FT-IR analysis. The authors acknowledge Cochin University of Science and Technology, Kochi for SEM analysis. This work did not receive any financial assistance.

\section{Compliance with ethical standards}

Conflict of interest All the authors of this manuscript would like to state that there is no conflict of interest.

Ethics approval The authors would like to declare that they have written the manuscript following the ethical standards.

\section{References}

1. Kuhnau J (1976) The flavonoids: a class of semi-essential food components: their role in human nutrition. World Rev Nutr Diet 24(1):117-191. https://doi.org/10.1159/000399407

2. Gopal JV (2013) Morin hydrate: botanical origin, pharmacological activity and its applications: a mini-review. J Pharmacogn 5(3):123-126. https://doi.org/10.1016/j.phcgj.2013.04.006

3. Lee KG, Shibamoto T, Takeoka GR, Lee SE, Kim JH, Park BS (2003) Inhibitoryeffects of plant-derived flavonoids and phenolic acids on malonaldehyde formation from ethylarachidonate. J Agric Food Chem 51(24):7203-7207. https://doi.org/10.1021/jf034 5447

4. Fang $\mathrm{SH}$, Hou YC, Chang WC, Hsiu SL, Chao PDL, Chiang BL (2003) Morin sulfates/glucuronides exert anti-inflammatory activity on activated macrophages and decreased the incidence of septic shock. Life Sci 74(6):743-756. https://doi.org/10.1016/j. Ifs.2003.07.017

5. Zeng LH, Wu J, Fung B, Tong JH, Mickle D, Wu TW (1997) Comparative protection against oxyradicals by three flavonoids on cultured endothelial cells. Biochem Cell Biol 75(6):717-720. https://doi.org/10.1139/o97-062

6. Wu TW, Fung KP, Zeng LH, Wu J, Hempel A, Grey A, Camerman N (1995) Molecular properties and myocardial salvage effects of morin hydrate. Biochem Pharmacol 49(4):537-543. https://doi. org/10.1016/0006-2952(94)00446-S

7. Nikolovska-Coleska Z, Klisarova LJ, Suturkova L, Dorevski K (1996) First and second derivative spectrophotometric determination of flavonoids chrysin and quercetin. Anal lett 29(1):97115. https://doi.org/10.1080/00032719608000395

8. Leposava P, Daniela D, Predrag D, Milena JS, Dusan M, Andrija C (2009) Spectrofluorimetric and HPLC determination of morin in human serum. Acta Chim Slov 56(4):967-972

9. Proestos C, Boziaris IS, Nychas GJ, Komaitis M (2006) Analysis of flavonoids and phenolic acids in Greek aromatic plants: investigation of their antioxidant capacity and antimicrobial activity. Food chem 95(4):664-671. https://doi.org/10.1016/j.foodc hem.2005.01.049

10. Fiamegos YC, Nanos CG, Vervoort J, Stalikas CD (2004) Analytical procedure for the in-vial derivatization-extraction of phenolic acids and flavonoids in methanolic and aqueous plant extracts followed by gas chromatography with mass-selective detection. J Chromatogr A 1041(1-2):11-18. https://doi.org/10.1016/j. chroma.2004.04.041

11. Zhang R, Zhang C, Zheng F, Li X, Sun CL, Chen W (2018) Nitrogen and sulfur co-doped graphene nanoribbons: a novel metal-free catalyst for high performance electrochemical detection of 2 , 4, 6-trinitrotoluene (TNT). Carbon 126(1):328-337. https://doi. org/10.1016/j.carbon.2017.10.042 
12. Masek A, Chrzescijanska E, Zaborski M (2014) Electrooxidation of morin hydrate at a Pt electrode studied by cyclic voltammetry. Food chem 148(1):18-23. https://doi.org/10.1016/j.foodc hem.2013.10.003

13. Tesio AY, Granero AM, Fernandez H, Zon MA (2011) Characterization of the surface redox process of adsorbed morin at glassy carbon electrodes. Electrochim acta 56(5):2321-2327. https:// doi.org/10.1016/j.electacta.2010.11.037

14. Temerk YM, Ibrahim MS, Kotb M (2011) Square-wave cathodic adsorptive stripping voltammetric determination of 3-hydroxyflavone, morin and hesperidin in bulk form and biological fluids in absence and presence of Cu (II). JBCS 22(11):2056-2064. https ://doi.org/10.1590/S0103-50532011001100006

15. Akshaya KB, Varghese A, Sudhakar YN, George L (2019) Electrocatalytic oxidation of morin on electrodeposited Ir-PEDOT nanograins. Food chem 270(1):78-85. https://doi.org/10.1016/j. foodchem.2018.07.074

16. Varghese A, Chitravathi S, Munichandraiah N (2016) Electrocatalytic oxidation and determination of morin at a poly $(2$, 5-dimercapto-1, 3, 4-thiadiazole) modified carbon fiber paper electrode. J Electrochem Soc 163(8):B471-B477. https://doi. org/10.1149/2.0021609jes

17. Yang L, Xu B, Ye H, Zhao F, Zeng B (2017) A novel quercetin electrochemical sensor based on molecularly imprinted poly (paraaminobenzoic acid) on 3D Pd nanoparticles-porous graphenecarbon nanotubes composite. Sens Actuators B 251(1):601-608. https://doi.org/10.1016/j.snb.2017.04.006

18. Yardım Y (2011) Cathodic adsorptive stripping voltammetry of abscisic acid using pencil-lead bismuth-film electrode. Rev Anal Chem 30(1):37-43. https://doi.org/10.1515/revac.2011.011

19. McCreery RL (2008) Advanced carbon electrode materials for molecular electrochemistry. Chem rev 108(7):2646-2687. https ://doi.org/10.1021/cr068076m

20. Aoki K, Okamoto T, Kaneko H, Nozaki K, Negishi A (1989) Applicability of graphite reinforcement carbon used as the lead of a mechanical pencil to voltammetric electrodes. J Electroanal Chem 263(2):323-331. https://doi.org/10.1016/00220728(89)85102-2

21. Purushothama HT, Nayaka YA, Vinay MM, Manjunatha $P$, Yathisha RO, Basavarajappa KV (2018) Pencil graphite electrode as an electrochemical sensor for the voltammetric determination of chlorpromazine. J Sci Adv Mater Devices 3(2):161-166. https:// doi.org/10.1016/j.jsamd.2018.03.007

22. David IG, Litescu SC, Popa DE, Buleandra M, lordache L, Albu C, Penu RL (2018) Voltammetric analysis of naringenin at a disposable pencil graphite electrode-application to polyphenol content determination in citrus juice. Anal Methods 10(48):57635772. https://doi.org/10.1039/C8AY02281J

23. Sharma S, Jain R, Raja AN (2019) Pencil graphite electrode: an emerging sensing material. J Electrochem Soc 167(3):037501037509. https://doi.org/10.1149/2.0012003JES

24. David IG, Popa DE, Buleandra M (2017) Pencil graphite electrodes: a versatile tool in electroanalysis. J anal methods chem 1(1):1-22. https://doi.org/10.1155/2017/1905968

25. Kausar A (2016) Electromagnetic interference shielding of polyaniline/poloxalene/carbon black composite. Int J Mater Chem 6(1):6-11. https://doi.org/10.5923/j.ijmc.20160601.02

26. Rahman MD, Kumar P, Park DS, Shim YB (2008) Electrochemical sensors based on organic conjugated polymers. Sensors 8(1):118-141. https://doi.org/10.3390/s8010118

27. Rokovic MK, Persi B, Mandic Z (2010) Electrochemical synthesis of polyaniline from aniline/cyclodextrin solutions. J Electroanal Chem 643(1-2):46-51. https://doi.org/10.1016/j.jelec hem.2010.03.010
28. Wang M, Fang G, Liu P, Zhou D, Ma C, Zhang D, Zhan J (2016) Fe304@ $3-C D$ nanocomposite as heterogeneous Fenton-like catalyst for enhanced degradation of 4-chlorophenol (4-CP). Appl Catal B Environ 188(1):113-122. https://doi.org/10.1016/j. apcatb.2016.01.071

29. Palanisamy $S$, Sakthinathan $S$, Chen $S M$, Thirumalraj B, Wu TH, Lou BS, Liu X (2016) Preparation of $\beta$-cyclodextrin entrapped graphite composite for sensitive detection of dopamine. Carbohydr Polym 135(1):267-273. https://doi.org/10.1016/j.carbp ol.2015.09.008

30. Nakahata M, Takashima Y, Yamaguchi H, Harada A (2011) Redoxresponsive self-healing materials formed from host-guest polymers. Nat Commun 2(1):1-6. https://doi.org/10.1038/ncomm S1521

31. Zheng L, Wu S, Lin X, Nie L, Rui L, Yang X (2002) Preparation and characterization of a novel $\beta$-cyclodextrin modified poly (N-acetylaniline) film. Macromolecules 35(16):6174-6177. https ://doi.org/10.1021/ma011663j

32. Rahemi V, Vandamme JJ, Garrido JMPJ, Borges F, Brett CMA, Garrido EMPJ (2012) Enhanced host-guest electrochemical recognition of herbicide MCPA using a $\beta$-cyclodextrin carbon nanotube sensor. Talanta 99(1):288-293. https://doi.org/10.1016/j.talan ta.2012.05.053

33. Meshkini A (2016) Acetone extract of almond hulls provides protection against oxidative damage and membrane protein degradation. J Acupunct Meridian Stud 9(3):134-142. https:// doi.org/10.1016/j.jams.2015.10.001

34. Joice EK, Varghese A, Sudhakar YN, Ganesh B, Selvaraj J (2018) Poly (aniline) decorated with nanocactus platinum on carbon fiber paper and its electrocatalytic behavior toward toluene oxidation. J Electrochem Soc 165(7):H399-H406. https://doi. org/10.1149/2.1121807jes

35. Joice EK, Rison S, Akshaya KB, Varghese A (2019) Platinum decorated polythiophene modified stainless steel for electrocatalytic oxidation of benzyl alcohol. J App Electrochem 49(9):937-947. https://doi.org/10.1007/s10800-019-01336-9

36. Luo Y, Hu Q, Liu G, Sun D (2015) Electrochemical behavior of ascorbic acid and rutin on poly (L-arginine)-graphene oxide modified electrode. Indian J Chem 53(1):187-192

37. Wang Q, Wang Y, Liu S, Wang L, Gao F, Gao F, Sun W (2012) Voltammetric detection of bisphenol a by a chitosan-graphene composite modified carbon ionic liquid electrode. Thin Solid Films 520(13):4459-4464. https://doi.org/10.1016/j. tsf.2012.02.069

38. Xiao P, Zhou Q, Xiao F, Zhao F, Zeng B (2006) Sensitive voltammetric determination of morin on a multi-walled carbon nanotubes-paraffin oil paste electrode. Int J Electrochem Sci 1(1):228-237

39. Sasikumar R, Govindasamy M, Chen SM, Chieh-Liu Y, Ranganathan P, Rwei SP (2017) Electrochemical determination of morin in Kiwi and Strawberry fruit samples using vanadium pentoxide nano-flakes. J Colloid Interface Sci 504(1):626-632. https://doi. org/10.1016/j.jcis.2017.03.039

40. Yola ML, Gupta VK, Eren T, Şen AE, Atar N (2014) A novel electro analytical nanosensor based on graphene oxide/silver nanoparticles for simultaneous determination of quercetin and morin. Electrochim Acta 120(1):204-211. https://doi.org/10.1016/j.elect acta.2013.12.086

Publisher's Note Springer Nature remains neutral with regard to jurisdictional claims in published maps and institutional affiliations. 